\title{
Capacitación y gestión del talento humano en administración de empresas
}

\section{Training and management of human talent in business administration}

\author{
María del Mar Hidalgo Cedeño \\ marimarhc 85@hotmail.com \\ Universidad Laica Eloy Alfaro de Manabï, Extensión Bahía de Caraquez \\ Ecuador \\ https://orcid.org/0000-0002-8899-0461 \\ Miguel Alberto Romero Zambrano \\ marz114@hotmail.com \\ Universidad Laica Eloy Alfaro de Manabï, Extensión Bahía de Caraquez \\ Ecuador \\ https://orcid.org/0000-0002-6990-0416 \\ Vicente Eduardo Pazmiño Chica \\ vicempaz@gmail.com \\ Universidad Laica Eloy Alfaro de Manabï, Extensión Bahía de Caraquez \\ Ecuador \\ https://orcid.org/0000-0002-0851-1214
}

Recibido: 10 de abril de 2019

Aprobado: 01 de junio de 2019

\begin{abstract}
RESUMEN
La investigación tuvo como objetivo determinar la efectividad de un programa de capacitación en gestión del talento humano dirigido a las cooperativas de transporte de taxi de la ciudad de Manta, Ecuador. Se aplicó un tipo de investigación explicativa. Para el análisis estadístico del pre y post test se aplicó la prueba T de Student. Entre las principales conclusiones se tiene que la efectividad del programa de capacitación fue significativa o positiva, lo que implica que las personas que participaron de la capacitación se encuentran en capacidad cognitiva para asumir con pertinencia y efectividad los procesos administrativos inherentes al desarrollo de las cooperativas de transporte en función de prestar un servicio de calidad en concordancia con una gerencia asertiva que puede contribuir a la articulación de permanencia en el tiempo de las cooperativa en base a una rentabilidad productiva con énfasis en la consolidación de políticas del buen vivir.
\end{abstract}


Descriptores: Calidad de la vida laboral; satisfacción en el trabajo; actitud laboral.

\section{ABSTRACT}

The objective of the research was to determine the effectiveness of a training program in human talent management aimed at taxi transport cooperatives in the city of Manta, Ecuador. A type of explanatory research was applied. For the statistical analysis of the pre and post test, the Student's T test was applied. Among the main conclusions is that the effectiveness of the training program was significant or positive, which implies that the people who participated in the training are in a cognitive capacity to assume with relevance and effectiveness the administrative processes inherent in the development of the cooperatives of transport based on providing a quality service in accordance with an assertive management that can contribute to the articulation of the cooperative's permanence in time based on productive profitability with emphasis on the consolidation of good living policies.

Descriptors: Quality of working life; Job satisfaction; Work attitudes

\section{INTRODUCCIÓN}

Las empresas cuentan con diversos patrimonios que le permiten trabajar en relación a los objetivos por los cuales han sido formadas, sin embargo; es de señalar que el principal bien con el que pueden contar es el recurso o talento humano, debido a que éste es el encargado de realizar, supervisar, entre otras responsabilidades, las operaciones que se realizan en la organización. Para lograrlo de forma eficaz y eficiente; la administración del talento humano tiene como tarea desarrollar habilidades y aptitudes del individuo, situación que conlleva a un proceso de capacitación permanente con el fin de generar aprendizajes en el talento humano que optimicen el funcionamiento de la empresa. Por lo tanto; se puede deducir que una organización que aprende es aquella que transfiere conocimientos a sus miembros, que construye un capital que no sólo se refleja en las cuentas de resultados de la empresa sino también en el potencial de sus miembros.

Partiendo de lo expuesto, Chiavenato (2009), propone que la gestión de talento humano, es un "equipo de personas que se ocupan de las actividades operativas para proporcionar asesoría interna en las áreas estratégicas tanto de la organización como de sus miembros" (p. 45), se puede señalar que para 
lograr un equipo de trabajo eficaz, debe entenderse que el talento humano no es solamente el esfuerzo o la actividad humana sino que también está integrada por otros factores que dan diversas modalidades a esta actividad. Entre estos factores se puede hacer mención de conocimientos, experiencias, motivación, intereses vocacionales, aptitudes, actitudes, habilidades, potencialidades, salud, todo esto ayuda a mejorar el desempeño de los empleados teniendo en cuenta que ellos son el pilar de la empresa.

Por tal motivo el talento humano, se ha convertido en la clave para el éxito organizacional, debido a que sin él se haría imposible innovar, enfrentar todas esas exigencias que se presentan en el entorno y el ambiente global de la organización. Pero desarrollar este talento es el principal reto que tienen que afrontar las empresas en la actualidad, para ello se crea la necesidad de incorporar una visión más integradora en la mente de los directivos, trabajadores de las diferentes compañías y siempre pensar que las estrategias que se utilicen deben implementarse en su totalidad para lograr esa concordancia que tanto se desea en el proceso organizacional.

En este sentido, las organizaciones se están desarrollando en un entorno que es muy variable, cambiante, que hace que estas se conviertan en poco tiempo vulnerables a dejar de ser competitivas y poniéndole una serie de trabas que impedirán mantenerse en el mercado. Por tal motivo es de vital importancia que las organizaciones por medio de una serie de incidencias $y / o$ acontecimientos enfrenten este tipo de situaciones, busquen alternativas para superarlas y lograr mantenerse bien posicionada en un futuro, logrando excelentes niveles de eficiencia, eficacia.

La gestión del talento humano puede señalarse como una actividad que depende menos de las jerarquías, órdenes, mandatos, destacándose la importancia de una participación activa de todos los trabajadores de la empresa como se ha hecho mención anteriormente, el objetivo es fomentar una relación de cooperación entre los directivos y los trabajadores para evitar los frecuentes enfrentamientos derivados de una relación jerárquica tradicional. 
Cuando la gestión del talento humano funciona correctamente, los empleados se comprometen con los objetivos a largo plazo de la organización, lo que permite que ésta se adapte mejor a los cambios en los mercados. Actualmente el mundo se encuentra en una constante transformación causada por el rápido avance tecnológico. Es por eso que las empresas han entendido la gran importancia de mantener actualizados a sus trabajadores. Así mismo; la globalización en la cual la humanidad se encuentra inmersa, ha puesto de manifiesto nuevas alternativas de administración empresarial enfocadas cada vez más en la gestión del talento humano, potencial que se debe desarrollar para poder competir en este creciente mercado.

Es así, que la visión con respecto a la gestión del talento humano ha cambiado; anteriormente se la veía como un gasto que la organización debía hacer, ahora los administradores exitosos han comprendido la gran importancia y beneficios que trae el contar con personal preparado dado que trae beneficios económicos, sociales, motivacionales, dentro de las mismas. En este sentido, el talento humano dentro de una organización tiende a tomar ciertas actitudes frente a su trabajo, por cuanto no solo se le debe proporcionar una retribución económica por parte de la misma sino también se debe tratar de satisfacer sus necesidades de crecimiento y desarrollo profesional.

Por lo tanto, la organización debe realizar gestiones centradas en el talento humano que sean dirigidas principalmente a la estabilidad y al mejor desenvolvimiento de todos los integrantes de la misma y una cultura organizacional de excelencia. En el proceso de gestión de talento humano intervienen todos los miembros activos de la empresa, entendiéndose por tales: la dirección general con tareas de mando, los asalariados con la negociación de un contrato, los representantes del personal. La gestión de talento humano se ha convertido en pilar estratégico en el desarrollo empresarial moderno. Prosiguiendo con lo expuesto; se puede señalar que cada día las personas constituyen una ventaja competitiva para la organización, y es por ello que la inversión en procesos de selección, formación, compensación, evaluación ha crecido en los últimos años. Si la organización cuenta con un talento humano 


\section{CIENCIAMATRIA}

Revista Interdisciplinaria de Humanidades, Educación, Ciencia y Tecnología

Año VI. Vol. VI. Nº10. Enero - Julio 2020

Hecho el depósito de ley: pp201602FA4721

ISSN-L: 2542-3029; ISSN: 2610-802X

Universidad Nacional Experimental Francisco de Miranda (UNEFM). Santa Ana de Coro. Venezuela

María del Mar Hidalgo Cedeño; Miguel Alberto Romero Zambrano; Vicente Eduardo Pazmiño Chica

preparado para asumir los retos que la competencia impone, entonces se puede hacer mención de la posibilidad de que la empresa pueda lograr sus objetivos en función del aporte significativo que realice el talento humano para lograrlo. Partiendo de lo expuesto, se tiene que las organizaciones cooperativas, deben tomar acciones para gestionar la calidad y productividad del talento humano que las conforman.

Por cuanto este tipo de organización empresarial, se convierten en una alternativa para muchos emprendedores que al tener una idea de negocio la gestionan bajo la figura jurídica de una cooperativa. En este sentido, Fernández (2010) señala que

Las organizaciones cooperativas se han convertido en actores relevantes del tejido productivo de las localidades, en virtud de los nuevos paradigmas del desarrollo, los cuales conceden protagonismo a las iniciativas que contribuyan a generar empleos y bienestar social, por lo que sus actuaciones deben responder a las necesidades locales e impactar favorablemente en su entorno. De tal manera, que son los asociados los encargados de realizar las acciones necesarias para el logro de estos objetivos (p. 47).

Se puede señalar que al talento humano en una cooperativa, se le conoce como "asociado"; situación que es importante destacar porque los asociados generalmente son las personas que fundan la organización y la llevan adelante, sin embargo esta situación no garantiza en la mayoría de los casos, que los asociados se encuentren en condiciones para administrarla y, sobre todo, gestionar del mejor modo posible el talento humano que se incorpora a laborar a la misma, quienes a la larga por condición de ley, terminan por convertirse en asociados.

Esto crea una complejidad que las personas encargadas de gerenciar la cooperativa y de administrar el talento humano deben asumir con diligencia a fin de no caer en errores que conlleven a pérdidas que paulatinamente ocasionen la quiebra o cierre de la cooperativa. Por el contrario, debe existir una formación o capacitación que permita que el talento humano que conforma la cooperativa pueda sentirse en condiciones de trabajo favorables, con el fin 


\section{CIENCIAMATRIA}

Revista Interdisciplinaria de Humanidades, Educación, Ciencia y Tecnología

Año VI. Vol. VI. Nº10. Enero - Julio 2020

Hecho el depósito de ley: pp201602FA4721

ISSN-L: 2542-3029; ISSN: 2610-802X

Universidad Nacional Experimental Francisco de Miranda (UNEFM). Santa Ana de Coro. Venezuela

María del Mar Hidalgo Cedeño; Miguel Alberto Romero Zambrano; Vicente Eduardo Pazmiño Chica

de incrementar la productividad de la cooperativa. En función de lo expuesto, Fernández (2010) señala que

Siendo el talento humano uno de los principales factores para alcanzar la competitividad, las organizaciones cooperativas deben convertirse en gestores del proceso de formación de sus asociados, atendiendo al quinto principio cooperativo y a lo contemplado en la Ley Especial de Asociaciones Cooperativas, donde se establece la obligación de destinar un 10\% como mínimo de los excedentes económicos al Fondo de Educación (p. 48).

Este principio pareciera que puede garantizar la formación del talento humano (asociados) que hacen vida en la cooperativa, situación que permite la posibilidad de que exista una capacitación constante en función de lograr elevar la productividad y ser cada día más competitivos. Las cooperativas deben entender que el talento humano es el principal capital con el que cuentan y que en la medida que lo capaciten en diversas competencias, más alta es la posibilidad de ser una organización exitosa. Sin embargo, Paredes (2003) citado por Fernández (2010) señala que "sin la educación permanente estas organizaciones fácilmente pueden desviarse por intereses económicos particulares o de otra índole, haciendo que no se consoliden y fracasen prontamente, generando en las comunidades escasa credibilidad hacia esta forma organizativa" (p. 49).

En este sentido, la capacitación del talento humano se hace necesaria y pertinente a fin de que las cooperativas se consoliden en sus servicios a lo largo del tiempo. Fernández (2010) señala que dentro de la formación que debe darse en las cooperativas es necesario la formación en administración, considera necesario incluir aspectos relacionados a la organización (71\%), administración del talento humano (71\%), control de gestión (57\%) y productividad y calidad (57\%), esto con la finalidad de evitar que las cooperativas trabajen de un modo que les conlleve ir a la quiebra parcial o total. Situación similar a la planteada por Fernández (2010), se evidencia en las cooperativas de transporte de taxi de Manta; donde por medio de un proceso de recopilación de información mediante entrevistas realizada por los 
investigadores, así como en la revisión de la rendición de cuentas realizadas en el ejercicio económico del año 2018, se ha podido evidenciar que existe desconocimiento de cómo administrar adecuadamente al talento humano, lo que da lugar a la generación de conflictos a nivel empresarial y social, por cuanto se originan pérdidas de recursos financieros, asociados desmotivados y desorientados en los diferentes puestos de trabajo, subestimando en muchos de los casos la capacidad de cada talento humano, originando un bajo rendimiento laboral, limitando así el cumplimiento de los objetivos empresariales cooperativos propuestos.

Por otro lado, se ha evidenciado la inexistencia de mecanismos técnicos para la contratación de personal, así como los procedimientos para el reclutamiento y selección, contratando personal no idóneo; así mismo la organización cooperativa, carece de planes de inducción, de capacitación y desarrollo, limitando, subestimando la capacidad de cada uno de los trabajadores, generando un bajo desempeño laboral. Esta falta de gestión del talento humano en las cooperativas de transporte de taxi de Manta, genera un ambiente de trabajo tenso, conflictos en el área de producción con un bajo desempeño laboral, y donde los asociados manifiestan que existe desconfianza de incompetencia por el cual no se desarrolla un sentido de progreso vivencial. Así mismo el incumplimiento de las metas establecidas, causan desmotivación en el personal, quienes en algunos casos, deciden abandonar la empresa, lo que implica un retraso en los procesos productivos y recarga de tareas para el personal restante. De no gestionar adecuadamente el talento humano en la organización, se pudiera generar una baja calidad en los productos terminados, así mismo el personal no se encontrará comprometido con las metas de la empresa cooperativa. Esto podría conllevar a altos costos de la mano de obra y la baja calificación del talento humano, con lo cual se corre el riesgo de que no aumente el desempeño laboral, lo cual perjudica a la cooperativa por cuanto podría haber demoras en la producción, lo que provocaría atrasos en la entrega de pedidos del producto a los clientes externos. 
Esta serie de inconvenientes puede producir distintos comportamientos y de alguna forma influir directamente en el día a día del talento humano (asociados) de la organización cooperativa, es por esta razón que la investigación está centralizada en gestionar un programa de capacitación en gestión del talento humano con el fin de promover en las cooperativas de transporte de taxi de Manta, un proceso formativo que les permita adecuarse a superar las problemáticas planteadas, en tal sentido, se plantea la siguiente interrogante de investigación: ¿Cuál es la efectividad de un programa de capacitación en gestión del talento humano dirigido a las cooperativas de transporte de taxi de la ciudad de Manta? Lo cual permite desarrollar el siguiente objetivo:

Determinar la efectividad de un programa de capacitación en gestión del talento humano dirigido a las cooperativas de transporte de taxi de la ciudad de Manta, Ecuador.

\section{DESARROLLO}

\section{Gestión del talento humano}

El talento humano puede señalarse como la persona que tiene las competencias necesarias para asumir un determinado cargo en la organización y ante lo cual, tiene la disposición de aprender cada día para mejorar su labor en función de ser productivo para la organización. Esto brinda la oportunidad de que se desarrolle el proceso conocido como "gestión del talento humano", en este sentido; Chiavenato (2009), establece que la gestión del talento humano está conformada por "las personas y las organizaciones. Las personas pasan gran parte de sus vidas trabajando en las organizaciones, las cuales dependen de individuos para operar y alcanzar el éxito" (p. 56). Partiendo de esta situación es de destacar, que el empleado juega un papel importante en el desarrollo de la empresa u organización, por cuanto se convierte en el factor central para que la misma alcance sus objetivos y metas propuestas.

Desde la visión de gestión del talento humano, se percibe al empleado como una persona que impulsa hacia el éxito a la organización, pero tal impulso debe 
estar acompañado de factores que incentiven en el talento humano, las condiciones necesarias para que tal impulso sea viable, cónsono, con la realidad que se pretende transformar en el ámbito organizacional. Es decir, la organización debe entender que el talento humano es su principal capital por cuanto éste es el encargado de gestionar la producción y calidad de los servicios que la empresa ofrece, en este sentido, se puede acotar, que el talento humano debe ser formado constantemente en diversas áreas con el fin de que se encuentran preparados integralmente y pueda así, asumir los retos que la organización propone para el cumplimiento de sus metas u objetivos.

En función de lo expuesto, se puede señalar que el contexto de la gestión del talento humano está conformado por las personas y las organizaciones. Las personas pasan gran parte de sus vidas trabajando en las organizaciones, las cuales dependen de las personas para operar y alcanzar el éxito. Las personas dependen de las organizaciones en que trabajan para alcanzar sus objetivos personales e individúales. Crecer en la vida y tener éxito casi siempre significa crecer dentro de las organizaciones. En este sentido, Carrión (2009), define a la gestión del talento humano como:

Un enfoque de gestión claramente estratégico cuyo objetivo es crear valor al accionista, al cliente, al empleado y a la sociedad. Para conseguirlo se centra en captar, desarrollar y retener al talento individual y organizado al servicio de la estrategia, los clientes, el mercado y el entorno (p. 428).

Se entiende que la empresa u organización tiene metas, para alcanzarlas debe trazar estrategias, planes, ante lo cual, el personal debe ser capacitado para que pueda actuar en beneficio del logro de tales metas, en este sentido, el talento humano es el encargado de gestionar los cambios, transformaciones, que han de cumplirse para que la empresa logre sus metas establecidas en función de obtener una alta productividad y calidad.

Partiendo de lo expuesto, la empresa u organización, deben gestionar en el talento humano capacidades que permitan que puedan fomentar aptitudes operativas en favor de las metas que se traza la empresa, de ahí que deba verse la capacitación permanente como parte de una inversión por cuanto un 
talento humano capacitado tiene mayores posibilidades de cumplir con éxito los planes trazados por la gerencia.

En función de lo expuesto, Alles (2009) plantea que el talento humano se percibe como "aquella serie de esfuerzos o actividades humanas que incluye conocimientos, experiencias, motivación, aptitudes, actitudes, habilidades y potencialidades de cada persona que integra a una organización" (p. 76), en consecuencia debe verse y entenderse como la posibilidad real que tiene la empresa se superarse cada día en función de la capacitación integral que reciba su talento humano en aras de que éste se encuentre en las mejores condiciones para asumir los retos que la organización se traza.

Prosiguiendo con lo expuesto, Alles (2009) plantea que "el trabajador debe de manera integral, estar integrado a la organización más allá de su mera actitud productiva en alguna forma, se busca la convicción del comportamiento de las personas en las organizaciones" (p. 77). En este sentido, la capacitación no solo debe entenderse como la preparación en habilidades técnicas, administrativas, personales, sino que debe entenderse como la oportunidad para gestionar convicción en el talento humano a fin de que se encuentre plenamente identificado con la empresa y con los planes que se proponen para alcanzar las metas.

Es decir, la gestión del talento humano debe verse como la posibilidad que tiene la organización de incentivar una aptitud pro activa para la consolidación de la visión y misión de la empresa. En este sentido, se hace necesario contar dentro de los planes de capacitación, aspectos que permitan fomentar el criterio reflexivo en el talento humano con el fin de incentivar un accionar que sea cónsono con las realidades a las cuales transita la empresa, es decir en búsqueda de productividad y calidad en los servicios que ofrece en satisfacción del cliente.

Po otro lado, Navarro (2009), señala que la gestión del talento en cualquier empresa es fundamental, "se ha convertido en una función clave antes, durante y después de cualquier crisis organizacional, en especial porque de manera fundamental son las personas el activo más importante de la organización sin 
tomar en cuenta el momento del ciclo económico" (p. 63). Desde lo planteado la persona alcanza el nivel más alto dentro de la empresa, situación que conlleva a prestar la debida atención para lograr así, una adecuada gestión del talento humano en la organización.

Es de destacar, que las organizaciones están conformada por personas y dependen de ellas para alcanzar sus objetivos y cumplir sus misiones. Para las personas, las organizaciones constituyen el medio de alcanzar varios objetivos personales en el mínimo tiempo, con el menor esfuerzo y conflicto. Es decir, se genera una sinergia de intereses entre la organización y el talento humano, de ese modo, ambos pueden conseguir sus objetivos en función del servicio que uno le preste al otro, situación que conlleva a un estado ganar - ganar entre la empresa y el talento humano con lo cual ambos se benefician en sus metas propuestas.

Esto conlleva a señalar que debe existir una motivación de parte de la gerencia hacia el talento humano para que éste se sienta estimulado al logro de las metas propuestas, pero a su vez el talento humano debe percibir que por medio del estímulo al trabajo va a conseguir sus metas individuales o personales para obtener un crecimiento económico - social; situación que conlleva a un trabajo productivo en función de alcanzar metas comunes.

En este sentido, se puede hacer mención que si la organización quiere alcanzar sus objetivos (crecimiento sostenido, rentabilidad, calidad en los productos y servicios, competitividad, entre otros.) de la mejor manera posible, debe saber canalizar los esfuerzos de las personas para que estas también alcancen sus objetivos individuales (mejores salarios, beneficios, estabilidad, satisfacción en el trabajo, oportunidad de crecimiento, entre otros.), de ese modo, se beneficien ambas partes.

La gestión del talento humano en las organizaciones es la función que permite la colaboración eficaz de las personas (empleados, funcionarios, recursos humanos o cualquier denominación utilizada) para alcanzar los objetivos organizacionales e individuales. La expresión administración de recursos humanos (ARH) todavía es la más común. Las personas pueden aumentar o 
disminuir las fortalezas y debilidades de una organización dependiendo de la manera como se trate.

En complemento, Chiavenato (2009) expone que: "cuando se habla de administración de recursos humanos, se toma como referencia la administración de personas que participan en las organizaciones, en las cuales desempeñan determinados roles" (p. 234). La administración de los recursos humanos busca construir y mantener un entorno de excelencia en la calidad para habilitar mejor a la fuerza de trabajo en la consecución de los objetivos de calidad y de desempeño operativo de la empresa.

\section{Capacitación del personal}

La capacitación del personal de una empresa permite que la misma se encuentre en condiciones de asumir retos que impulsen los niveles de productividad y calidad, con lo cual, la organización puede transitar hacia la excelencia. De ese modo, Hill (2009) señala que:

La capacitación es una actividad sistemática, planificada y permanente cuyo propósito general es preparar, desarrollar e integrar a los recursos humanos al proceso productivo, mediante la entrega de conocimientos, desarrollo de habilidades y actitudes necesarias para el mejor desempeño de todos los individuos en sus actuales y futuros cargos y adaptarlos a las exigencias cambiantes del entorno (p. 329).

En este sentido, la gerencia debe tener entre sus planes de crecimiento, programas de capacitación hacia el talento humano con el fin de que este pueda adquirir competencias que le permitan incentivar la productividad de la empresa. Por lo tanto, capacitación se puede entender como el perfeccionamiento técnico de las personas para que se desempeñen eficientemente en las funciones asignadas, y así solucionar anticipadamente problemas potenciales dentro de la organización o empresa. A través de la capacitación es posible adecuar el perfil del talento humano, conocimientos, habilidades y actitudes requerido en un puesto de trabajo. En este sentido, García (2009), expresa que: 


\section{CIENCIAMATRIA}

Revista Interdisciplinaria de Humanidades, Educación, Ciencia y Tecnología

Año VI. Vol. VI. №10. Enero - Julio 2020

Hecho el depósito de ley: pp201602FA4721

ISSN-L: 2542-3029; ISSN: 2610-802X

Universidad Nacional Experimental Francisco de Miranda (UNEFM). Santa Ana de Coro. Venezuela

María del Mar Hidalgo Cedeño; Miguel Alberto Romero Zambrano; Vicente Eduardo Pazmiño Chica

Los planes de capacitación ayudan a las personas a desempeñar su trabajo actual y los beneficios de ésta pueden extenderse a toda su vida laboral o profesional, además pueden ayudarlos a desarrollar habilidades para responsabilidades futuras, dichos planes cuenta con objetivos muy claros, entre los cuales se pueden mencionar: Conducir a la empresa a una mayor rentabilidad y a los empleados a tener una actitud más positiva, mejorando el conocimiento del puesto a todos los niveles. Ayudando al personal a identificarse con los objetivos de la empresa, fomentando la autenticidad, la apertura y la confianza (p. 210).

La capacitación del talento humano representa una vía para alcanzar el éxito individual y colectivo en la empresa, en este sentido, ninguna organización debe dejar de lado la capacitación de su talento humano, debe incorporar en su presupuesto lo necesario para atender a sus empleados por medio un crecimiento que sea producto de la capacitación. Partiendo de lo expuesto, García (2009):

Los beneficios de la capacitación no son sólo para el trabajador, sino también para la empresa; ya que para ambos constituye la mejor inversión para enfrentar los retos del futuro. Destacando beneficios como: un trabajo preparado para el manejo de conflictos, la toma de decisiones y la solución de problemas mediante herramientas técnicamente probadas; aumentando así la confianza del individuo (p. 230).

En fin, la capacitación es de suma importancia para inculcar en el talento humano, las competencias necesarias para que puedan tener un óptimo desempeño laboral en la medida que alcanzan un crecimiento personal mediante la obtención de metas personales y organizacionales. Es de destacar, que se puede dar una capacitación conocida como general y otra como específica, en relación a esto se plantea lo siguiente:

- Capacitación general: es sobre lo que debe saber el empleado de la empresa y que hacer para que la misma funcione cada día mejor.

- Capacitación específica: que debe saber el empleado sobre su cargo y área de trabajo para desempeñarse mejor 
Cómo puede observarse, de acuerdo a lo planteado por García (2009) existen dos clasificaciones de capacitación en la empresa, ambas persiguen el mismo objetivo que es la de mejorar el conocimiento y habilidades del talento humano, solo que una lo hace en modo general y la otra en particular; de ese modo, la gerencia puede programar planes de capacitación de modo grupal, donde participen todo el talento humano, y capacitación donde participe un grupo específico de talento humano a fin de optimizar una determinada área de la empresa.

Desde otra perspectiva, Frigo (2011) define a la capacitación como: "Capacitación, o desarrollo de personal, es toda actividad realizada en una organización, respondiendo a sus necesidades, que busca mejorar la actitud, conocimiento, habilidades o conductas de su personal" (p. 1). Por lo tanto, la capacitación se convierte en un fenómeno que procura mejorar a la organización en razón que lo hace su talento humano, de allí se pueden generar acciones que sean favorables a la productividad y calidad de la empresa. En este sentido, Frigo (2011) señala que concretamente, la capacitación:

- Busca perfeccionar al colaborador en su puesto de trabajo,

- En función de las necesidades de la empresa,

- En un proceso estructurado con metas bien definidas.

Prosiguiendo con lo expuesto por Frigo, la necesidad de capacitación surge cuando hay diferencia entre lo que una persona debería saber para desempeñar una tarea, y lo que sabe realmente. Estas diferencias suelen ser descubiertas al hacer evaluaciones de desempeño, o descripciones de perfil de puesto. Dados los cambios continuos en la actividad de las organizaciones, prácticamente ya no existen puestos de trabajo estáticos. Cada persona debe estar preparada para ocupar las funciones que requiera la empresa. En este sentido, la empresa debe estar atenta a los cambios que surgen en el mercado para incorporarlos a la organización mediante un proceso de capacitación y entrenamiento continuo de su personal con el fin de que pueda brindarle respuestas favorables a las exigencias planteadas por los clientes. Esta 
situación permite que la empresa u organización tenga en la capacitación una de las mejores inversiones que puede realizar, debido que por medio de este proceso puede mantener actualizado al personal en consonancia con los estándares exigidos en el mercado, situación que conlleva a mantener y aumentar la cartera de clientes de la empresa y con ello elevar su productividad.

\section{METODOLOGÍA DE LA INVESTIGACIÓN}

El trabajo realizado se basó en un tipo de investigación explicativa (Hernández Sampieri, Fernández Collado \& Baptista 2014), la definen "como aquellos estudios que van más allá de la descripción de los fenómenos", con la finalidad de Determinar la efectividad de un programa de capacitación en gestión del talento humano dirigido a las cooperativas de transporte de taxi de la ciudad de Manta, Ecuador, apoyándose en un diseño cuasi experimental de pre prueba, postprueba, con grupo control. Para el análisis estadístico del pre y post test se aplicó la prueba $\mathrm{T}$ de Student. Construyéndose las siguientes hipótesis: Nula HO: No existe diferencias significantes entre ambos momentos, lo que es igual no funcionó el tratamiento. Alterna $\mathrm{H} 1$ : Existen diferencias significantes entre ambos momentos, lo que es igual, sí funcionó el tratamiento. La población objeto de estudio estuvo conformada por 62 personas que laboran en las cooperativas de transporte de la ciudad de Manta, la muestra que participó en el grupo experimental (tratamiento) fue de 31 personas quienes conformaron el grupo RG1, mientras que otros 27 conformaron el grupo control o RG2 (no se les aplicó tratamiento), trabajándose del siguiente modo:

- $\quad$ RG1 = Grupo al cual se le aplicó el tratamiento

- 01 = Momento de la pre prueba del RG1

- $\quad X=$ Tratamiento aplicado a RG1

- 02 = Momento de la post prueba a RG1

- $\quad$ RG2 = Grupo al cual no se le aplicó el tratamiento

- 03 = Momento de la pre prueba del RG2

- $\quad$ - = Tratamiento no aplicado a RG2 
- $\quad 04$ = Momento de la post prueba a RG2

$\begin{array}{llll}\text { RG1 } & 01 & X & 02 \\ \text { RG2 } & 03 & - & 04\end{array}$

De acuerdo al diagrama anterior, la pre prueba se aplicó a los dos grupos, luego se procedió a aplicar el tratamiento a RG1, el cual tuvo una duración de seis semanas, una semana posterior a la finalización del tratamiento se procedió a aplicar la post prueba a ambos grupos nuevamente, en este sentido, se puede esquematizar el proceso de la investigación, del siguiente modo:

1. Inicio del proceso de capacitación y aplicación del pre test a ambos grupos (experimental y control)

2. Cierre del programa se aplicó la post prueba a ambos grupos

\section{RESULTADOS DE LA INVESTIGACIÓN}

Se procedió a aplicar una prueba de hipótesis utilizando un nivel de significancia de $5 \%$. Las hipótesis planteadas son:

Nula H0: No existe diferencias significantes entre ambos grupos, lo que es igual no funcionó el tratamiento.

Alterna $\mathrm{H} 1$ : Existen diferencias significantes entre ambos grupos, lo que es igual, sí funcionó el tratamiento.

La tabla de "pruebas independientes" (pre test), el valor de Levene es $<0,05$ entonces se asumen varianzas iguales. En dichos resultados el valor de significancia bilateral fue de 0,768 el cual es $>0,05$, lo que indica que no existen diferencias significativas entre ambos grupos (experimental y control) para el momento del pre test.

La prueba independiente referente al pos test, el valor de Levene también fue de < 0,05 , por lo tanto, se asumen varianzas iguales. En este caso, el nivel de significancia bilateral cambió a "0", lo que es lo mismo < 0,05, lo que indica diferencias significativas en ambos grupos (experimental y control).

El tratamiento aplicado al grupo experimental surtió efectos positivos por cuanto existió diferencia estadística entre el momento inicial (sin tratamiento) y el momento final (una vez aplicado el tratamiento), se rechaza $\mathrm{H} 0$ y se acepta $\mathrm{H} 1$, lo que indica que el tratamiento aplicado fue favorable. 


\section{CONCLUSIONES}

De acuerdo a los resultados, la efectividad de un programa de capacitación en gestión del talento humano dirigido a las cooperativas de transporte de taxi de la ciudad de Manta, Ecuador, fue significativa o positiva, lo que implica que las personas que participaron de la capacitación se encuentran en capacidad cognitiva para asumir con pertinencia y efectividad los procesos administrativos inherentes al desarrollo de las cooperativas de transporte en función de prestar un servicio de calidad en concordancia con una gerencia asertiva que puede contribuir a la articulación de permanencia en el tiempo de las cooperativa en base a una rentabilidad productiva con énfasis en la consolidación de políticas del buen vivir, lo cual concuerda con Pruna Jacome \& Albán Yánez (2019), quienes afirman que es necesario capacitar a las personas que trabajan en la prestación de servicio en el sector turismo con la finalidad de estar acordes a trabajar en brindar un servicio de calidad al cliente, siendo Manta una ciudad turística se hace factible la realización de este tipo de investigación.

Por otro lado, el tratamiento aplicado procuro fomentar competencias en cuanto a relaciones interpersonales con la finalidad de promover un clima organizacional acorde a la prestación de un servicio efectivo al cliente, siendo necesario promover la empatía, siendo esta descrita por Aldana \& Piña (2018), como aquella capacidad que permite atender a los clientes en equidad de condiciones, promoviéndose un servicio sinérgico como factor de calidad al cliente.

\section{REFERENCIAS CONSULTADAS}

1. Aldana, J., \& Piña, J. (2018). Calidad del servicio prestado al cliente por los instructores de gimnasios. Revista Arbitrada Interdisciplinaria Koinonía, 2(3), 172-197. Recuperado de http://fundacionkoinonia.com.ve/ojs/index.php/revistakoinonia/article/view $/ 59 / 46$

2. Alles, M. (2009). Desarrollo del Talento Humano Basado en competencias. Ciudad Buenos Aires AR., Granica.

3. Carrión, J. (2009). Estrategia: de la visión a la acción. 2da edición. ESIC 
Editorial. Madrid.

4. Chiavenato, I. (2009). Gestión del talento humano. 3ra Edición. Mc Graw Hill/ Interamericana Editores S. A. De C. V. México.

5. Fernández (2010). Formación del talento humano: factor clave para el desarrollo de organizaciones cooperativas. Recuperado de http://revistas.luz.edu.ve/index.php/mc/article/viewFile/11736/11368

6. Frigo, E. (2011). ¿Qué es la capacitación? Documento en línea. Recuperado de http://www.forodeseguridad.com/artic/rrhh/7011.htm

7. García (2009). El proceso de capacitación, sus etapas e implementación para mejorar el desempeño del recurso humano en las organizaciones. Recuperado de http://www.eumed.net/ce/2011b/imgl.html

8. Hernández Sampieri, R., Fernández Collado , C., \& Baptista , L. (2014). Metodología de la investigación . México: Editorial Mc - Graw - Hill Interamericana. Sexta edición.

9. Hill, M (2009). Formación, Capacitación, Desarrollo de RR.HH y su Importancia en las Organizaciones. México McGraw-Hill

10.Navarro, E. (2009). Quieres Salvar Tu Empresa. Editorial Planeta (gestión 2000).

11.Pruna Jacome, L., \& Albán Yánez, C. (2019). Estudio del Talento Humano en las prácticas de los centros de información turística y su incidencia en el turismo de la provincia de Cotopaxi. Revista Arbitrada Interdisciplinaria Koinonía, 4(8), 451-467. doi:http://dx.doi.org/10.35381/r.k.v4i8.294

\section{REFERENCES CONSULTED}

1. Aldana, J., \& Piña, J. (2018). Quality of service provided to the client by gym instructors. Interdisciplinary Arbitrated Review Koinonía, 2 (3), 172197. http://fundacionkoinonia.com.ve/ojs/index.php/revistakoinonia/article/view $\underline{159 / 46}$

2. Alles, M. (2009). Development of Human Talent Based on competencies. City Buenos Aires AR., Granica.

3. Carrión, J. (2009). Strategy: from vision to action. 2nd edition. ESIC 
Editorial. Madrid.

4. Chiavenato, I. (2009). Human talent management. 3rd edition. Mc Graw Hill / Interamericana Editores S. A. De C. V. México.

5. Fernández (2010). Training of human talent: key factor for the development of cooperative organizations. Retrieved from http://revistas.luz.edu.ve/index.php/mc/article/viewFile/11736/11368

6. Frigo, E. (2011). What is the training? Online document Retrieved from http://www.forodeseguridad.com/artic/rrhh/7011.htm

7. Garcia (2009). The training process, its stages and implementation to improve the performance of human resources in organizations. Recovered from http://www.eumed.net/ce/2011b/jmgl.html

8. Hernández Sampieri, R., Fernández Collado, C., \& Baptista, L. (2014). Investigation methodology . Mexico: Editorial Mc - Graw - Interamerican Hill. Sixth edition.

9. Hill, M (2009). Training, Training, HR Development and its Importance in Organizations. Mexico McGraw-Hill

10. Navarro, E. (2009). You want to save your company. Editorial Planeta (management 2000).

11.Pruna Jacome, L., \& Albán Yánez, C. (2019). Study of Human Talent in the practices of tourist information centers and their impact on tourism in the province of Cotopaxi. Interdisciplinary Arbitrated Review Koinonía, 4 (8), 451-467. doi: http: //dx.doi.org/10.35381/r.k.v4i8.294 